\title{
Oscillation for a class of coupled van der Pol oscillators with delays
}

\author{
Chunhua Feng ${ }^{a^{*}}$, Yuming Wei ${ }^{b}$ \\ College of Mathematics and Statistics, Guangxi Normal University, Guilin, China, 541004 \\ a chfeng@mailbox.gxnu.edu.cn, ${ }^{\mathrm{b}}$ ymwei@mailbox.gxnu.edu.cn \\ *corresponding author
}

Keywords: Coupled van der Pol model, Delay, Oscillation.

\begin{abstract}
In this paper, a class of coupled van der Pol oscillators with time delays is investigated. By means of Chafee's criterion, some sufficient conditions to guarantee the existence of oscillatory solutions for the model are obtained. Computer simulations are provided to demonstrate the proposed result
\end{abstract}

\section{Introduction}

The dynamical behaviour of coupled oscillators with or without time delays have been studied by many researchers [1-19]. For example, Zhang and Gu have investigated the coupled van der Pol oscillators with two delays:

$$
\begin{aligned}
& x_{1}^{\prime \prime}(t)+\varepsilon\left(x_{1}^{2}(t)-1\right) x_{1}^{\prime}(t)+x_{1}(t)=\alpha\left[y_{1}\left(t-\tau_{2}\right)-x_{1}(t)\right], \\
& y_{1}^{\prime \prime}(t)+\varepsilon\left(y_{1}^{2}(t)-1\right) y_{1}^{\prime}(t)+y_{1}(t)=\alpha\left[x_{1}\left(t-\tau_{1}\right)-y_{1}(t)\right] .
\end{aligned}
$$

By using the theory of normal form and the center manifold theorem, the explicit expression for determining the direction of the Hopf bifurcation and the stability of the bifurcating periodic solution were derived. Barron et al. have considered the stability and corrective behaviour of a ring of coupled van der Pol oscillators [2, 3]:

$$
\begin{aligned}
& x_{1}^{\prime}(t)+\alpha\left(x_{1}^{2}(t)-1\right) x_{1}^{\prime}(t)+x_{1}(t)=b_{1}\left[x_{n}(t)-2 x_{1}(t)+x_{2}(t)\right] \text {, } \\
& x_{2}^{\prime}(t)+\alpha\left(x_{2}^{2}(t)-1\right) x_{2}^{\prime}(t)+x_{2}(t)=b_{2}\left[x_{1}(t)-2 x_{2}(t)+x_{3}(t)\right] \text {, } \\
& x_{n}^{\prime}(t)+\alpha\left(x_{n}^{2}(t)-1\right) x_{n}^{\prime}(t)+x_{n}(t)=b_{n}\left[x_{n-1}(t)-2 x_{n}(t)+x_{1}(t)\right] \text {. }
\end{aligned}
$$

Where $b_{i}$ is the coupling parameter corresponding to the ith oscillator. If $\theta_{i}$ is the angular position of the $i$ th oscillator, then $\theta_{i}=i \Delta \theta$, where $\Delta \theta=2 u /(n-1)$. The stability analysis is based on the transformation of the linearized equation of the ring into a canonical Hill equation. The collective behaviour is numerically studied. Three values of the oscillators constant are assumed. Wang and Chen have discussed a ring of coupled van der Pol oscillators with time delay coupling as follows:

$$
\begin{aligned}
& u_{1}^{\prime \prime}(t)+\left(\alpha-\beta u_{1}^{2}(t)\right) u_{1}^{\prime}(t)+a u_{1}(t)=A u_{2}^{\prime}(t-\tau), \\
& u_{2}^{\prime \prime}(t)+\left(\alpha-\beta u_{2}^{2}(t)\right) u_{2}^{\prime}(t)+a u_{2}(t)=A u_{3}^{\prime}(t-\tau), \\
& \cdots \cdots \cdots \cdots \cdots \cdots \cdots \cdots \cdots \cdots \cdots \cdots \cdots \cdots \cdots \cdots \cdots \cdots \cdots \cdots \cdots \cdots \cdots \cdots \cdots \cdots \cdots \cdots \cdots \cdots \cdots \\
& u_{n}^{\prime \prime}(t)+\left(\alpha-\beta u_{n}^{2}(t)\right) u_{n}^{\prime}(t)+a u_{n}(t)=A u_{1}^{\prime}(t-\tau) .
\end{aligned}
$$

Where $A$ is the coupling strength, and $\tau$ is the time delay. By using the method of multiple scales, the amplitude equations are obtained. Chosen $A$ and $\tau$ as the bifurcation parameters, the dynamical 
behaviour arising from the bifurcation is classified. Motivated by the above models, in this paper we consider the following general coupled van der Pol system with delays

$$
\begin{aligned}
& u_{1}^{\prime \prime}(t)+\varepsilon_{1}\left(u_{1}^{2}(t)-a_{1}\right) u_{1}^{\prime}(t)+c_{1} u_{1}(t)=b_{1}\left[u_{n}\left(t-s_{n}\right)-2 u_{1}\left(t-s_{1}\right)+u_{2}\left(t-c_{2}\right)\right], \\
& u_{2}^{\prime \prime}(t)+\varepsilon_{2}\left(u_{2}^{2}(t)-a_{2}\right) u_{2}^{\prime}(t)+c_{2} u_{2}(t)=b_{2}\left[u_{1}\left(t-s_{1}\right)-2 u_{2}\left(t-s_{2}\right)+u_{3}\left(t-c_{3}\right)\right] \text {, } \\
& u_{n}^{\prime \prime}(t)+\varepsilon_{n}\left(u_{n}^{2}(t)-a_{n}\right) u_{n}^{\prime}(t)+c_{n} u_{n}(t)=b_{n}\left[u_{n-1}\left(t-s_{n-1}\right)-2 u_{n}\left(t-s_{n}\right)+u_{1}\left(t-c_{1}\right)\right] .
\end{aligned}
$$

where $\varepsilon_{i}, a_{i}, b_{i}$, and $c_{i}$ are constants, time delays $s_{i} \geq 0(i=1,2, \ldots, n)$. Our goal is to investigate the dynamic behaviour of $n$ coupled oscillators. Based on the Chafee's criterion: if a time delay system has a unique unstable equilibrium point, and all solutions of the system are bounded, then this system will generate a limit cycle, namely a periodic solution [20]. Some existent results in the literature have been extended.

\section{Preliminaries}

It is convenient to write equation (4) as an equivalent $2 n$ dimensional first order equation:

$$
\begin{aligned}
& x_{1}^{\prime}(t)=x_{2}(t), \\
& x_{2}^{\prime}(t)=\varepsilon_{1} a_{1} x_{2}(t)-\varepsilon_{1} x_{1}^{2}(t) x_{2}(t)-c_{1} x_{1}(t)+b_{1}\left[x_{2 n-1}\left(t-\tau_{2 n-1}\right)-2 x_{1}\left(t-\tau_{1}\right)+x_{3}\left(t-\tau_{3}\right)\right], \\
& \ldots \ldots \ldots \ldots \ldots \ldots \ldots \ldots \ldots \ldots \ldots \ldots \ldots \ldots \ldots \ldots \ldots \ldots \ldots \ldots \ldots \ldots \ldots \ldots \ldots \ldots \ldots \ldots \ldots \ldots \ldots \ldots \ldots \ldots \ldots \ldots \ldots \ldots \ldots \ldots \ldots \ldots \ldots \ldots \ldots \ldots \ldots \ldots \ldots \ldots \ldots \ldots \ldots \\
& x_{2 n}^{\prime}(t)=\varepsilon_{n} a_{n} x_{2 n}(t)-\varepsilon_{n} x_{2 n-1}^{2}(t) x_{2 n}(t)-c_{n} x_{2 n-1}(t)+b_{n}\left[x_{2 n-3}\left(t-\tau_{2 n-1}\right)-2 x_{2 n-1}\left(t-\tau_{1}\right)+x_{1}\left(t-\tau_{1}\right)\right] .
\end{aligned}
$$

where time delays $s_{i}=\tau_{2 i-1}(i=1,2, \ldots, n)$. Obviously, the origin $x_{i}=0(i=1,2, \ldots, 2 n)$ is an equilibrium point of equation (5). The linearization of the coupled equation (5) at origin is

$$
\begin{aligned}
& x_{1}^{\prime}(t)=x_{2}(t) \\
& x_{2}^{\prime}(t)=\varepsilon_{1} a_{1} x_{2}(t)-c_{1} x_{1}(t)+b_{1}\left[x_{2 n-1}\left(t-\tau_{2 n-1}\right)-2 x_{1}\left(t-\tau_{1}\right)+x_{3}\left(t-\tau_{3}\right)\right], \\
& \ldots \ldots \ldots \ldots \ldots \ldots \ldots \ldots \ldots \ldots \ldots \ldots \ldots \ldots \ldots \ldots \ldots \ldots \ldots \ldots \ldots \ldots \ldots \ldots \ldots \ldots \ldots \ldots \ldots \ldots \ldots \ldots \ldots \ldots \ldots \ldots \ldots \ldots \ldots \ldots \ldots \ldots \ldots \ldots \ldots \ldots \ldots \ldots \ldots \ldots \\
& x_{2 n-1}^{\prime}(t)=x_{2 n}(t) \\
& x_{2 n}^{\prime}(t)=\varepsilon_{n} a_{n} x_{2 n}(t)-c_{n} x_{2 n-1}(t)+b_{n}\left[x_{2 n-3}\left(t-\tau_{2 n-1}\right)-2 x_{2 n-1}\left(t-\tau_{1}\right)+x_{1}\left(t-\tau_{1}\right)\right] .
\end{aligned}
$$

The equations (5) and (6) can be expressed in the following matrix form (7) and (8) respectively:

$$
\begin{aligned}
& X^{\prime}(t)=P X(t)+Q X(t-\tau)+F(X(t)) \\
& X^{\prime}(t)=P X(t)+Q X(t-\tau)
\end{aligned}
$$

where $X(t)=\left[x_{1}(t), x_{2}(t), \ldots, x_{2 n}(t)\right]^{T}, X(t-\tau)=\left[x_{1}\left(t-\tau_{1}\right), 0, x_{3}\left(t-\tau_{3}\right), 0, \ldots, x_{2 n-1}\left(t-\tau_{2 n-1}\right), 0\right]^{T}$. Both $p=\left(p_{i j}\right)_{2 n \times 2 n}$ an $\mathrm{d} Q=\left(q_{i j}\right)_{2 n \times 2 n}$ are $2 n \times 2 n$ matrices.

Lemma 1 Assume that the matrix $R(P+Q)$ is a nonsingular matrix, then equation (6) has a unique equilibrium point, implying that equation (5) has a unique equilibrium point.

Proof An equilibrium point $x^{*}=\left[x_{1}^{*}, x_{2}^{*}, \ldots, x_{2 n}^{*}\right]^{T}$ of equation (6) is a constant solution of the following algebraic equation: 


$$
\begin{aligned}
& x_{2}^{*}=0, \\
& \varepsilon_{1} a_{1} x_{2}^{*}-c_{1} x_{1}^{*}+b_{1}\left[x_{2 n-1}^{*}-2 x_{1}^{*}+x_{3}^{*}\right]=0, \\
& \cdots \cdots \cdots \cdots \\
& \varepsilon_{n} a_{n} x_{2 n}^{*}-c_{n} x_{2 n-1}^{*}+b_{n}\left[x_{2 n-3}^{*}-2 x_{2 n-1}^{*}+x_{1}^{*}\right]=0 .
\end{aligned}
$$

The matrix form of (9) is the following equation:

$$
P x^{*}+Q x^{*}=(P+Q) x^{*}=R x^{*}=0
$$

Noting that $R$ is a $2 n \times 2 n$ matrix. According to the linear algebraic knowledge, if $R$ is a nonsingular matrix, equation (9) has only one solution, namely, the trivial solution, implying that equation (6) has a unique equilibrium point. For equation (5), the nonlinear terms $\varepsilon_{i} x_{2 i-1}^{2}(t) x_{2 i}(t) \quad(i=1,2, \ldots, n)$ are high order infinitesimal when $x_{2 i-1}$ and $x_{2 i}(i=1,2, \ldots, n)$ tend to zero. Therefore, equation (6) has a unique equilibrium point suggests that equation (5) has a unique equilibrium point.

Lemma 2 If parameters $\varepsilon_{i}(i=1,2, \ldots, n)$ are positive values, then all solutions of equation (5) are bounded.

Proof To prove the boundedness of the solutions in equation (5), we construct a Lyapunov function $V(t)=0.5 \sum_{i=1}^{2 n} x_{i}^{2}(t)$. Calculating the derivative of $V(t)$ through equation (5) one get

$$
\begin{aligned}
& \left.V^{\prime}(t)\right|_{(5)}=\sum_{i=1}^{2 n} x_{i}(t) x_{i}^{\prime}(t)=x_{1}(t) x_{2}(t)+x_{2}(t)\left\{\varepsilon_{1} a_{1} x_{2}(t)+\cdots+b_{1} x_{3}\left(t-\tau_{3}\right)\right\} \\
& \quad+\cdots+x_{2 n-1}(t) x_{2 n}(t)+x_{2 n}(t)\left\{\varepsilon_{n} a_{n} x_{2 n}(t)+\cdots+b_{n}\left[x_{2 n-3}\left(t-\tau_{2 n-3}\right)-\cdots+x_{1}\left(t-\tau_{1}\right)\right\}\right. \\
& =-\varepsilon_{1} x_{1}^{2}(t) x_{2}^{2}(t)-\varepsilon_{2} x_{3}^{2}(t) x_{4}^{2}(t)-\cdots-\varepsilon_{n} x_{2 n-1}^{2}(t) x_{2 n}^{2}(t)+\left(1-c_{1}\right) x_{1}(t) x_{2}(t) \\
& \quad+b_{n} x_{2 n}(t)\left[x_{2 n-3}\left(t-\tau_{2 n-3}\right)-2 x_{2 n-1}\left(t-\tau_{2 n-1}\right)+x_{1}\left(t-\tau_{1}\right)\right]
\end{aligned}
$$

Noting that as $x_{2 i-1} x_{2 i}(i=1,2, \ldots, n)$. tend to infinity, $x_{2 i-1}^{2} x_{2 i}^{2} \quad(i=1,2, \ldots, n)$. are higher order infinity than $x_{2 i-1} x_{2 i}(i=1,2, \ldots, n)$. Since $0<\varepsilon_{i}(i=1,2, \ldots, n)$, so there exists suitably large $M>0$ such that $\left.V^{\prime}(t)\right|_{(5)}<0$ as $\left|x_{i}\right| \geq M(i=1,2, \ldots, 2 n)$. This means that all solutions of equation (5) are bounded.

\section{Main Results}

In order to guarantee the boundedness of the solutions, in the following we always select parameter values $\varepsilon_{i}>0(i=1,2, \ldots, n)$.

Theorem 1 Assume that linearized equation (6) has a unique equilibrium point for selected parameter values. Let $\alpha_{1}, \alpha_{2}, \ldots, \alpha_{2 n}$ be $2 n$ eigenvalues of matrix $p$. If there exists some $\alpha_{k}, k \in\{1,2, \ldots, 2 n\}$. such that $\alpha_{k}>0$ or the real part $\operatorname{Re}\left(\alpha_{k}\right)>0$ then the unique equilibrium point of equation (5) is unstable, implying that equation (5) generates a limit cycle, namely, an oscillatory solution.

Proof In order to avoid unnecessary complexity, we restrict ourselves to the case of equation (6) as $\tau_{2 i-1}=\tau_{*}(1,2, \ldots, n)$, where $\tau_{*}=\operatorname{Min}\left\{\tau_{1}, \tau_{3}, \ldots, \tau_{2 n-1}\right\}$. . From equation (8) we have the form 


$$
X^{\prime}(t)=P X(t)+Q X\left(t-\tau_{*}\right)
$$

Assume that $\beta_{1}, \beta_{2}, \ldots, \beta_{2 n}$ are $2 n$ eigenvalues of matrix $Q$. Since $\alpha_{1}, \alpha_{2}, \ldots, \alpha_{2 n}$ are $2 n$ eigenvalues of matrix $P$, then the characteristic equation corresponding to equation (12) is the following:

$$
\prod_{i=1}^{2 n}\left(\lambda-\alpha_{i}-\beta_{i} e^{-\lambda \tau_{*}}\right)=0
$$

Noting that there are $n$ row elements are zeros in matrix $Q$. Therefore, there is at least one eigenvalue, say $\beta_{k}=0$. We are led to an investigation of the nature of the roots for the equation

$$
\lambda-\alpha_{k}-\beta_{k} e^{-\lambda \tau_{*}}=\lambda-\alpha_{k}=0
$$

The characteristic equation (14) indicates that $\lambda=\alpha_{k}>0$. if $\alpha_{k}>0$, or $\lambda=\operatorname{Re}\left(\alpha_{k}\right)>0$ if $\operatorname{Re}\left(\alpha_{k}\right)>0$ In other words, there exists a positive or a positive real part eigenvalue of characteristic equation (14). Hence, the trivial solution of equation (12) is unstable. Noting that in a time delay system, as the value of delay increases, the instability of the trivial solution still maintains. So for any values of time delays $\tau_{2 i-1} \geq \tau_{*}(1,2, \ldots, n)$, the trivial solution of equation (8) (or equation (6)) is unstable. Recall that in equation (5), the nonlinear terms $\varepsilon_{i} x_{2 i-1}^{2}(t) x_{2 i}(t) \quad(i=1,2, \ldots, n)$ are higher order infinitesimal when $x_{2 i-1}(t)$ and $x_{2 i}(t)(i=1,2, \ldots, n)$ ten to zero. Therefore, the uniqueness and instability of the equilibrium point of equation (6) implies that equation (5) has a unique equilibrium point which is unstable. This means that equation (5) generates a limit cycle, namely, an oscillatory solution of equation (5) [20].

We adopt the following measure and norm of matrices [21]: $u(P)=\max _{1 \leq i \leq n}\left\{1+\varepsilon_{i}-c_{i}\right\}$, and $\|Q\|=\max _{1 \leq i \leq n}\left\{\sum_{i=1}^{n}\left|q_{i j}\right|\right\}$.

Theorem 2 Assume that linearized equation (6) has a unique equilibrium point for selected parameter values. Suppose that the following condition holds

$$
(|| Q \|) e \tau_{*} \exp \left(-\tau_{*}|\mu(P)|\right)>1
$$

then the unique equilibrium point of equation (5) is unstable, implying that equation (5) generates a limit cycle, namely, an oscillatory solution.

Proof We still consider equation (12). Let $z(t)=\sum_{i=1}^{2 n}\left|x_{i}(t)\right|$, then we have

$$
z^{\prime}(t) \leq \mu(P) z(t)+\|Q\| z\left(t-\tau_{*}\right)
$$

Specially, for equation

$$
y^{\prime}(t)=\mu(P) y(t)+\|Q\| y\left(t-\tau_{*}\right)
$$


If the unique equilibrium point of equation (17) is stable, then the characteristic equation associated with equation (17) given by

$$
\lambda=\mu(P)+\|Q\| \exp \left(-\lambda \tau_{*}\right)
$$

will have a real negative root say $\lambda_{0}$, and we have from (18)

$$
\begin{aligned}
& \left|\lambda_{0}\right|+|\mu(P)| \geq\|Q\| \exp \left(-\lambda_{0} \tau_{*}\right)=\|Q\| \exp \left(\left|\lambda_{0}\right| \tau_{*}\right) \\
= & \left.\|Q\| \exp \left[\left|\lambda_{0}\right|+|\mu(P)|\right) \tau_{*}\right] \exp \left(-|\mu(P)| \tau_{*}\right)
\end{aligned}
$$

Using formula $\exp \left(\left|\lambda_{0}\right|+|\mu(P)|\right) \tau_{*} \geq e\left(\left|\lambda_{0}\right|+|\mu(P)|\right) \tau_{*} \quad$ one can obtain

$$
1 \geq\|Q\| e \tau_{*} \exp \left(-\tau_{*}|\mu(P)|\right)
$$

Inequality (20) contradicts (15). Hence, our claim regarding the instability of the trivial solution of equation (17) is valid. According to the comparison principle of differential equation we have $z(t) \leq y(t)$. Since the trivial solution of equation (17) is unstable, implying that the trivial solution of equation (16) is unstable. By Chafee’s limit cycle criterion, equation (5) generates an oscillatory solution.

\section{Simulation Results}

Example 1 First we consider a four coupled van der Pol equation:

$$
\begin{aligned}
x_{1}^{\prime}(t) & =x_{2}(t), \\
x_{2}^{\prime}(t) & =\varepsilon_{1} a_{1} x_{2}(t)-\varepsilon_{1} x_{1}^{2}(t) x_{2}(t)-c_{1} x_{1}(t)+b_{1}\left[x_{7}\left(t-\tau_{7}\right)-2 x_{1}\left(t-\tau_{1}\right)+x_{3}\left(t-\tau_{3}\right)\right], \\
x_{3}^{\prime}(t) & =x_{4}(t), \\
x_{4}^{\prime}(t) & =\varepsilon_{2} a_{2} x_{4}(t)-\varepsilon_{2} x_{3}^{2}(t) x_{4}(t)-c_{2} x_{3}(t)+b_{2}\left[x_{1}\left(t-\tau_{1}\right)-2 x_{3}\left(t-\tau_{3}\right)+x_{5}\left(t-\tau_{5}\right)\right], \\
x_{5}^{\prime}(t) & =x_{6}(t), \\
x_{6}^{\prime}(t) & =\varepsilon_{3} a_{3} x_{6}(t)-\varepsilon_{3} x_{5}^{2}(t) x_{6}(t)-c_{3} x_{5}(t)+b_{3}\left[x_{3}\left(t-\tau_{3}\right)-2 x_{5}\left(t-\tau_{5}\right)+x_{7}\left(t-\tau_{7}\right)\right], \\
x_{7}^{\prime}(t) & =x_{8}(t), \\
x_{8}^{\prime}(t) & =\varepsilon_{4} a_{4} x_{8}(t)-\varepsilon_{4} x_{7}^{2}(t) x_{8}(t)-c_{4} x_{7}(t)+b_{4}\left[x_{5}\left(t-\tau_{5}\right)-2 x_{7}\left(t-\tau_{7}\right)+x_{1}\left(t-\tau_{1}\right)\right] .
\end{aligned}
$$

We fix $\varepsilon_{1}=0.0045, \varepsilon_{2}=0.0075, \varepsilon_{3}=0.005, \varepsilon_{4}=0.0065$, and first select time delays as 3.5,3.6,3.8, and 3.2 The other parameter values are: $a_{1}=1.15, a_{2}=1.25, a_{3}=1.24, a_{4}=1.32$; $b_{1}=0.18, b_{2}=0.22, b_{3}=0.28, b_{4}=0.32 ; c_{1}=1.56, c_{2}=1.45, c_{3}=1.36, c_{4}=1.25$. Corresponding to equation (21), the eigenvalues of matrix $P 0.0025 \pm 1.2490 i, 0.0030 \pm 1.2042 i, 0.0040 \pm 1.1662 i$, and $0.0045 \pm 1.1180 \mathrm{i}$ Since there are eigenvalues of matrix $P$ have positive real parts, it is easy to check the condition of Theorem 1 are satisfied. Equation (21) generates an oscillatory solution (see Fig.1). In order to see the effect of time delays, we increase the time delays as 6.5,6.6, 6.8, and 6.2 , the dynamical behaviour still maintains. However, the oscillatory amplitude and frequency both are changed (see Fig.2).

Example 2 Then we consider a five coupled van der Pol equation: 


$$
\begin{aligned}
& x_{1}^{\prime}(t)=x_{2}(t), \\
& x_{2}^{\prime}(t)=\varepsilon_{1} a_{1} x_{2}(t)-\varepsilon_{1} x_{1}^{2}(t) x_{2}(t)-c_{1} x_{1}(t)+b_{1}\left[x_{9}\left(t-\tau_{9}\right)-2 x_{1}\left(t-\tau_{1}\right)+x_{3}\left(t-\tau_{3}\right)\right], \\
& x_{3}^{\prime}(t)=x_{4}(t), \\
& x_{4}^{\prime}(t)=\varepsilon_{2} a_{2} x_{4}(t)-\varepsilon_{2} x_{3}^{2}(t) x_{4}(t)-c_{2} x_{3}(t)+b_{2}\left[x_{1}\left(t-\tau_{1}\right)-2 x_{3}\left(t-\tau_{3}\right)+x_{5}\left(t-\tau_{5}\right)\right], \\
& x_{5}^{\prime}(t)=x_{6}(t), \\
& x_{6}^{\prime}(t)=\varepsilon_{3} a_{3} x_{6}(t)-\varepsilon_{3} x_{5}^{2}(t) x_{6}(t)-c_{3} x_{5}(t)+b_{3}\left[x_{3}\left(t-\tau_{3}\right)-2 x_{5}\left(t-\tau_{5}\right)+x_{7}\left(t-\tau_{7}\right)\right], \\
& x_{7}^{\prime}(t)=x_{8}(t), \\
& x_{8}^{\prime}(t)=\varepsilon_{4} a_{4} x_{8}(t)-\varepsilon_{4} x_{7}^{2}(t) x_{8}(t)-c_{4} x_{7}(t)+b_{4}\left[x_{5}\left(t-\tau_{5}\right)-2 x_{7}\left(t-\tau_{7}\right)+x_{9}\left(t-\tau_{9}\right)\right], \\
& x_{9}^{\prime}(t)=x_{10}(t), \\
& x_{10}^{\prime}(t)=\varepsilon_{5} a_{5} x_{10}(t)-\varepsilon_{5} x_{9}^{2}(t) x_{10}(t)-c_{5} x_{9}(t)+b_{5}\left[x_{7}\left(t-\tau_{7}\right)-2 x_{9}\left(t-\tau_{9}\right)+x_{1}\left(t-\tau_{1}\right)\right] .
\end{aligned}
$$

We fix $\varepsilon_{1}=0.00055, \varepsilon_{2}=0.00065, \varepsilon_{3}=0.00075, \varepsilon_{4}=0.00045$, and $\varepsilon_{5}=0.00036$. Select time delays as $0.6,0.8,0.2$ and 0.4 . The other parameter values are $a_{1}=1.55, a_{2}=1.65, a_{3}=1.25, a_{4}=1.35, a_{5}=1.45 ; b_{1}=0.25, b_{2}=0.35, b_{3}=0.45, b_{4}=0.55, b_{5}=0.65$; $\mathrm{c}_{1}=0.62, c_{2}=0.52, c_{3}=0.42, c_{4}=0.22, c_{5}=0.32$.

Based on the selected parameter values, corresponding to equation (22) we have $u(P)=1.0009$ $\|Q\|=2.2$, and $\tau_{*}=0.2$. Then $(\|Q\|) e \tau_{*} \exp \left(-\tau_{*}|u(P)|\right)=4.8474>1$. The condition (15) is satisfied. Equation (22) generates an oscillatory solution (see Fig.3).

\section{Acknowledgements}

This work was supported by National Natural Science Foundation of China (11361010).

\section{References}

[1] Zhang, J.M. and Gu, X.S. (2010) Stability and bifurcation analysis in the delay-coupled van der Pol oscillators. Applied Mathematical Modelling, 34, 2291-2299.

[2] Barron, M.A., Medina, D.Y. and Hilerio, I. (2014) Collective behavior of a ring of van der Pol oscillators under Gaussian and random coupling schemes. Open Journal of Applied Sciences, 4, 383-391.

[3] Barron, M.A. (2016) Stability of a ring of coupled van der Pol oscillators with non-uniform distribution of the coupling parameters. Journal of Applied Research and Technology, 14, 62-66.

[4] Wang, W.Y. and Chen, L.J. (2015) Weak and non-resonant double Hopf bifurcations in $m$ coupled van der Pol oscillators with delay coupling. Applied Mathematical Modelling, 39, 30943102.

[5] Shen, Z.L. and Zhan, C.R. (2014) Double Hopf bifurcation of coupled dissipative StuartLandau oscillators with delay. Applied Mathematics and Computation, 227, 553-566.

[6] Jallouli, A., Kacem, N. and Bouhaddi, N. (2017) Stabilization of solutions in coupled nonlinear pendulums with simultaneous external and parametric excitations. Commun Nonlinear Sci Numer Simulat, 42, 1-11.

[7] Maccari, A. (2012) Response control for the externally excited van der Pol oscillator with nonlocal feedback. Journal of Sound and Vibration, 331, 987-995. 
[8] Rajeev, R., Govind, M. and Balaram, B. (2016) Effect of external force on the dynamics of nonlinearly coupled self excited oscillators. Procedia Engineering, 144, 1007-1014.

[9] Qian, Y.H. and Chen, S.M. (2010) Accurate approximate analytical solutions for multi-degreeof-freedom coupled van der Pol-Duffing oscillators by homotopy analysis method. Commun Nonlinear Sci Numer Simulat, 15, 3113-3130.

[10]Das, S. and Maharatna, K. (2013) Fractional dynamical model for the generation of ECG like signals from filtered coupled van-der Pol oscillators. Computer Methods and Programs in Biomedicine, 112, 490-507.

[11]Ghosh, D., Chowdhury, A.R. and Saha, P. (2008) On the various kinds of synchronization in delayed Duffing-Van der Pol system. Commun Nonlinear Sci Numer Simulat, 13, 790-803.

[12] Yuan, Y.C., Xue, H.X. and Tang, W.Y. (2017) An improved time domain coupled model of cross-flow and in-line vortex induced vibration for flexible risers. Ocean Engineering, 136, 117-128.

[13]Cveticanin, L. (2014) Coupled Van der Pol oscillator with non-integer order connection. Commun Nonlinear Sci Numer Simulat, 19, 3362-3373.

[14]Joshi, S.K., Sen, S. and Kar, I.N. (2016) Synchronization of coupled oscillator dynamics. IFAC-PAPERSONLINE, 49, 320-325.

[15]Wirkus, S. and Rand, R. (2002) The dynamics of two coupled van der Pol oscillators with delay coupling. Nonlinear Dynamics, 30, 205-221.

[16]Theotokoglou, E.E. and Panayotounakos, D.E. (2017) Nonlinear asymptotic analysis of a system of two free coupled oscillators with cubic nonlinearities. Applied Mathematical Modelling, 42, 509-520.

[17]Ersoz, E.K., Desroches, Krupa, M. (2017) Synchronization of weakly coupled canard oscillators. Physica D: Nonlinear Phenomena, 349, 46-61.

[18]Narahara, K. (2017) Mutual synchronization of oscillating pulse edges in point-coupled transmission lines with regularly spaced tunel diodes. Commun Nonlinear Sci Numer Simulat, 42, 236-246.

[19]Wiggers, V. and Rech, P.C. (2017) Multistability and organization of periodicity in a van der Pol-Duffing oscillator. Chaos, Solitons \& Fractals, 103, 632-637.

[20]Chafee, N. (1971) A bifurcation problem for a functional differential equation of finitely retarded type, Journal of Mathematical Analysis and Applications, 35, 312-348.

[21]Gopalsamy, K. (1992) Stability and oscillations in delay differential equations of population dynamics. Kluwer Academic Publishers 
Fig. 1 Oscillation of the solutions, delays: $(3.5,3.6,3.8,3.2)$.

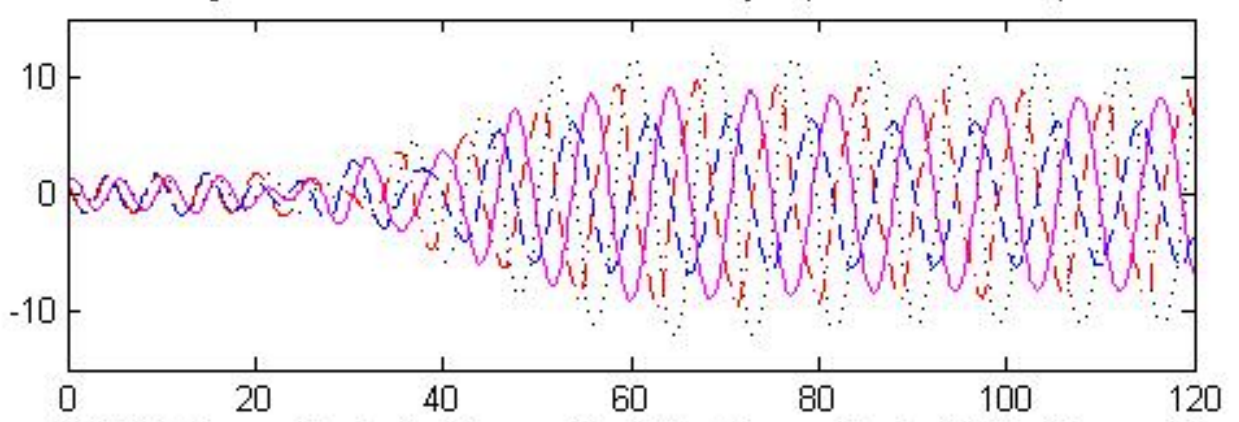

(a) Solid line: $x_{1}(t)$, dashed line: $x_{2}(t)$, dotted line: $x_{3}(t)$, dashdotted line: $x_{4}(t)$.

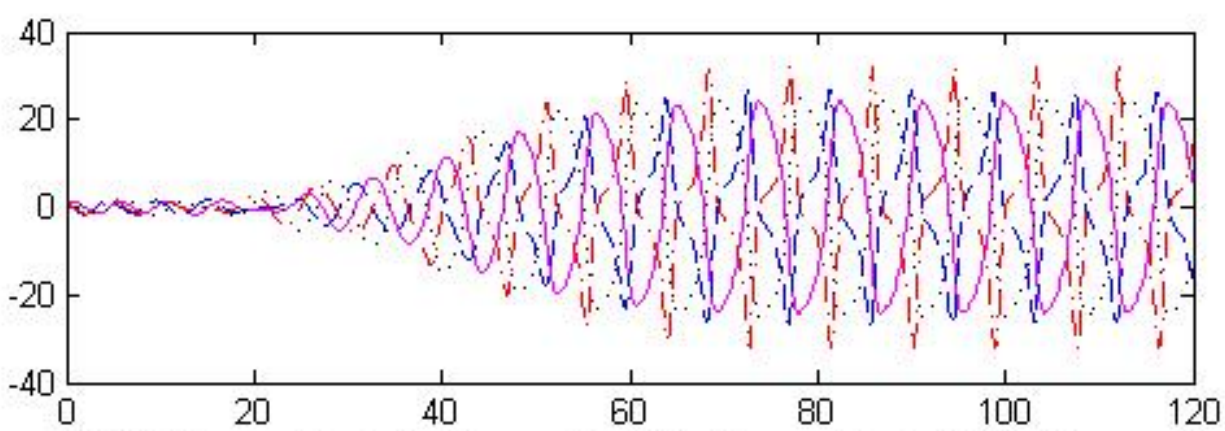

(b) Solid line: $x_{5}(t)$, dashed line: $x_{6}(t)$, dotted line: $x_{7}(t)$, dashdotted line: $x_{8}(t)$.

Fig.2 Oscillation of the solutions, delays: $(6.5,6.6,6.8,6.2)$.

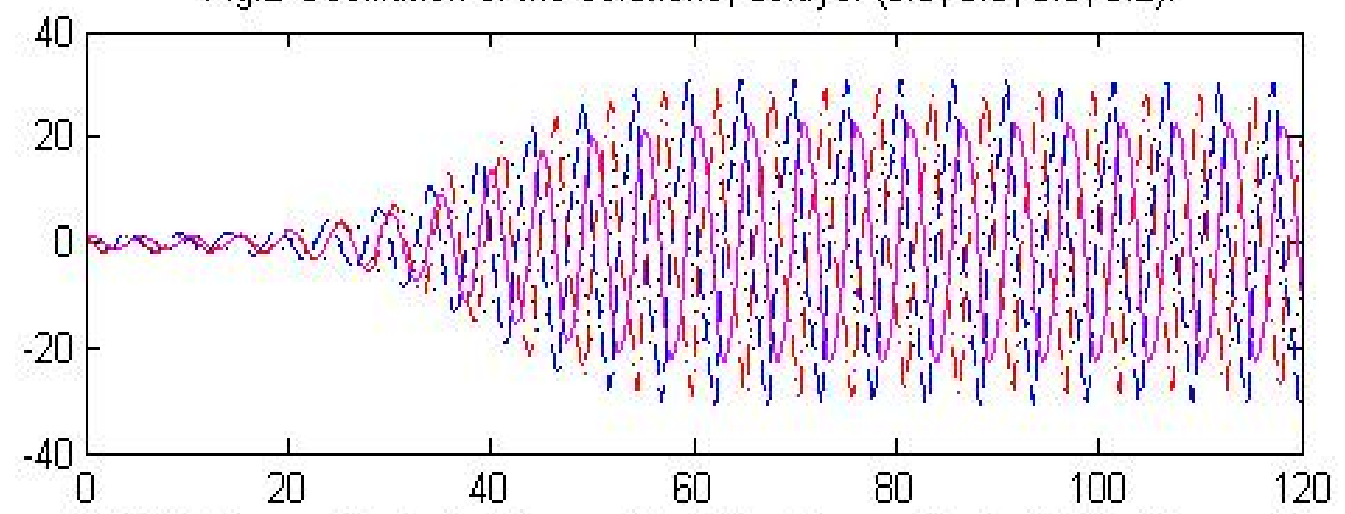

(a) Solid line: $x_{1}(t)$, dashed line: $x_{2}(t)$, dotted line: $x_{3}(t)$, dashdotted line: $x_{4}(t)$.

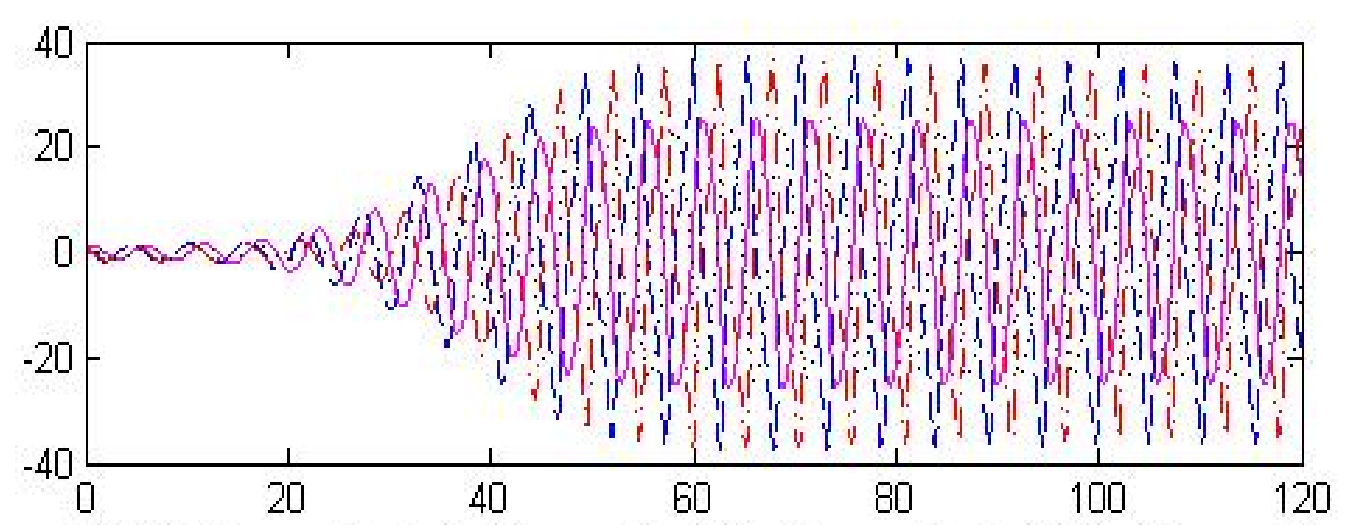

(b) Solid line: $x_{5}(t)$, dashed line: $x_{6}(t)$, dotted line: $x_{7}(t)$, dashdotted line: $x_{8}(t)$. 
Fig.3 Oscillation of the solutions, delays: $(0.5,0.6,0.8,0.2,0.4)$.

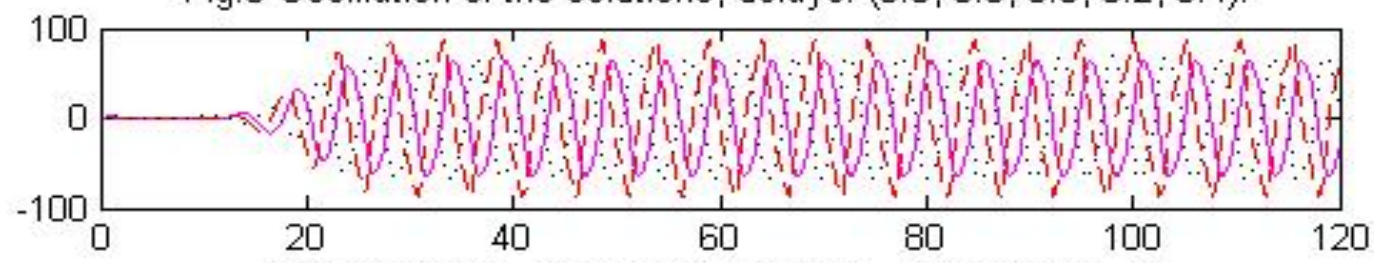

(a) Solid line: $x_{1}(t)$, dashed line: $x_{2}(t)$, dotted line: $x_{3}(t)$.

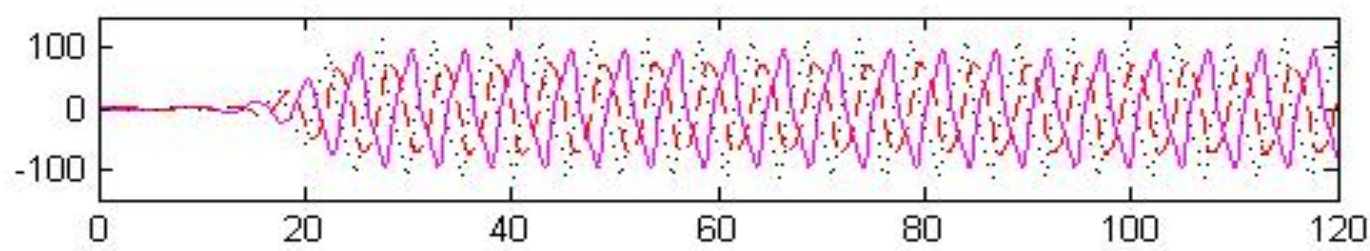

(b) Solid line: $x_{4}(t)$, dashed line: $x_{5}(t)$, dotted line: $x_{6}(t)$.

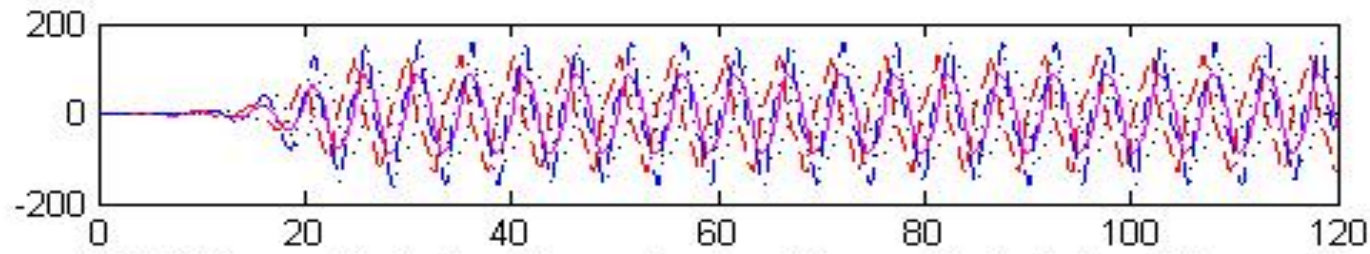

(c) Solid line: $x_{7}(t)$, dashed line: $x_{8}(t)$, dotted line: $x_{9}(t)$, dashdotted line: $x_{10}(t)$. 